\title{
EFFECT OF ENVIRONMENTS ON GEOCAST ROUTING PROTOCOLS
}

\author{
Mohamed M. Zahra \\ Electrical Engineering Department, Faculty of Engineering, AL-AZHAR \\ University Cairo, Egypt
}

(Received July 17, 2011 Accepted September 22, 2011)

This paper considers the problem of providing a geocast service in mobile Ad hoc networks. It figures out how to select parameters for two selected Geocast routing protocols via simulation. It presents a study for the effect of simulation environments (node density, traffic, mobility) on geocast routing protocols. Geocast is useful for sending messages to everyone in a specified geographical region.

\section{1- INTRODUCTION}

Mobile Ad hoc NETwork (MANET) consists of wireless mobile hosts that communicate with each other in the absence of a fixed infrastructure. The ability to establish an Ad hoc network without using a fixed infrastructure makes them useful in many scenarios, including disaster recovery, search-and rescue in remote areas, and home networking applications. Thus MANET has attracted extensive research interests over the past several years.

Especial scenarios like public safety or battle field require reliable Multicast (voice, data...) communication in which the leader must send the same information to more than one destination. Such multicasting is often used, because it is more efficient than multiple unicasts in terms of the communication costs. Cost considerations are all the more important for a MANET because the mobile hosts communicate with each other over wireless links. In MANET environments, the multicast problem is complex because network topology change may be frequent.

This paper considers a variation of multicasting, namely, geocasting. Thus, a geocast is delivered to a set of nodes within a specified geographical area. Also this paper will refer to the specified area as the "geocast region". A set of nodes in the geocast region forms the geocast group. If a host resides within a geocast region at a given time, it automatically becomes a member of the corresponding geocast group at that time. To determine a group membership, each node is required to know its own physical location, i.e., its precise geographic coordinates, which may be obtained using the Global Positioning System (GPS).

Geocast routing protocols of a MANET can be classified into three categories: Flooding-based, cluster-based and routing-based protocols. Flooding-based protocols use Flooding or a variant of Flooding to forward geocast packets from a source to a geocast region. Protocols in this category include the Location-Based Multicast (LBM) protocol [1] and the Voronoi diagram based geocasting protocol [2]. Cluster-based protocols geographically partition a MANET into several disjointed and equally sized cellular regions and select a cluster head in each region for executing information exchange. Protocols in this category include GeoGRID [3], and Obstacle-Free 
Single/Multi- Destination Geocasting Protocols. For more details on these protocols, see [4]. Routing-based protocols create routes from the sending source to the requested geocast region via control packets. Protocols in this category include the Mesh-based Geocast Routing Protocol (MGRP) [5], Geocast Adaptive Mesh Environment for Routing protocol (GAMER) [6] and GeoTORA protocol [7].

\section{2- GEOCAST ROUTING PROTOCOLS FOR MANET}

In this study, four case studies for the effect of node density, traffic, mobility, combination effect on LBM (for flooding-based protocols), GeoGRID (for clusterbased protocols) and GAMER (for the routing-based protocol category) are performed.

\section{2-1 Flooding Based Geocast Protocol (LBM)}

LBM algorithms are essentially identical to multicast flooding, with a modification that a node which is not in the forwarding zone does not forward a multicast packet to its neighbors. Thus, implementing LBM schemes requires that a node that can determine if it is in the forwarding zone for a particular multicast packet. Two algorithms are presented, LBM-box and LBM-step.

LBM-box: This algorithm uses a forwarding zone that is rectangular in shape (refer to Figure 1) It defines the forwarding zone to be the smallest rectangle that includes current location of sender $\mathrm{S}$ and the multicast region, in that the sides of the rectangle are parallel to the $\mathrm{X}$ (horizontal) and $\mathrm{Y}$ (vertical) axes. In Figure 1a, the multicast region is the rectangle whose corners are $\mathrm{P}, \mathrm{O} \mathrm{B}$ and $\mathrm{Q}$, and the forwarding zone is the rectangle whose corners are $\mathrm{S}, \mathrm{A}, \mathrm{B}$ and $\mathrm{C}$.

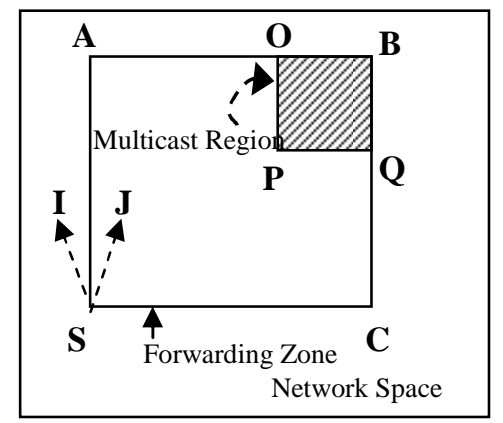

(a) Source node outside the multicast region

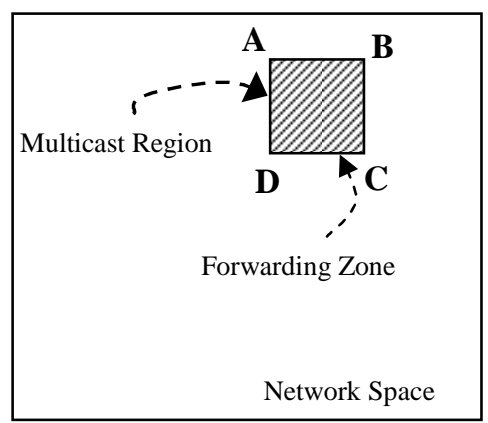

(b) Source node within the multicast region

Figure 1: Location Based Multicast scheme 1(LBM-box)

Note that the size of a rectangular forwarding zone above is dependent on: (i) size of the multicast region and (ii) location of the sender. To provide additional control on the size of the forwarding zone, we define a parameter $\delta$, which can be used to extend the forwarding zone. When $\delta$ is positive, the rectangular forwarding zone is extended in positive and negative $\mathrm{X}$ and $\mathrm{Y}$ directions by $\delta$ (thus each side increases by $2 \delta)$.

LBM-step: without including the forwarding zone explicitly, node $\mathrm{S}$ includes three pieces of information with its multicast packet:

- The multicast region specification. 
- The location of the geometrical center, $\left(\mathrm{X}_{\mathrm{C}}, \mathrm{Y}_{\mathrm{C}}\right)$ of the multicast region. The distance of any node $\mathrm{Z}$ from $\left(\mathrm{X}_{\mathrm{C}}, \mathrm{Y}_{\mathrm{C}}\right)$ will be denoted as $\mathrm{DIST}_{\mathrm{Z}}$ in the rest of this discussion.

- The coordinates of sender $\mathrm{S},\left(\mathrm{X}_{\mathrm{S}}, \mathrm{Y}_{\mathrm{S}}\right)$.

When a node $I$ receives the multicast packet from node $S$, this node I determines if it belongs to the multicast region. If this node $\mathrm{I}$ is in multicast region, it accepts the multicast packet, and then calculates its distance from location $\left(\mathrm{X}_{\mathrm{C}}, \mathrm{Y}_{\mathrm{C}}\right)$, denoted as DIST $_{i}$, for some parameter $\delta$.

- IF DISTS $+\delta \geq$ DIST $_{\mathrm{i}}$, then node I forwards the packet to its neighbors. Before forwarding the multicast packet, node $\mathrm{I}$ replaces the $\left(\mathrm{X}_{\mathrm{S}}, \mathrm{Y}_{\mathrm{S}}\right)$ coordinates received in the multicast packet by its own coordinates $\left(\mathrm{X}_{i}, \mathrm{Y}_{i}\right)$.

* Else DIST $\mathrm{S}+\delta<\mathrm{DIST}_{\mathrm{i}}$, in this case, node I sees whether or not sender $\mathrm{S}$ is within the multicast region. If $S$ is in the multicast region, then node I forwards the packet to its neighbors, otherwise, I discards the packet.

\section{2-2 GeoGRID, Cluster-Based Geocast Protocol}

In this protocol the geographic area of the MANET is partitioned into 2D logical grid as illustrated in Figure 2. Each cell in a grid is a square of size $\mathrm{d} \times \mathrm{d}$. The cells are numbered $(\mathrm{x}$, y) following the conventional $x-y$ coordinate. Each host still has a unique ID (such as IPaddress). In each cell one host will be elected as the gateway of the cell. The responsibility of gateway hosts is to propagate geocast packets to neighboring cells.

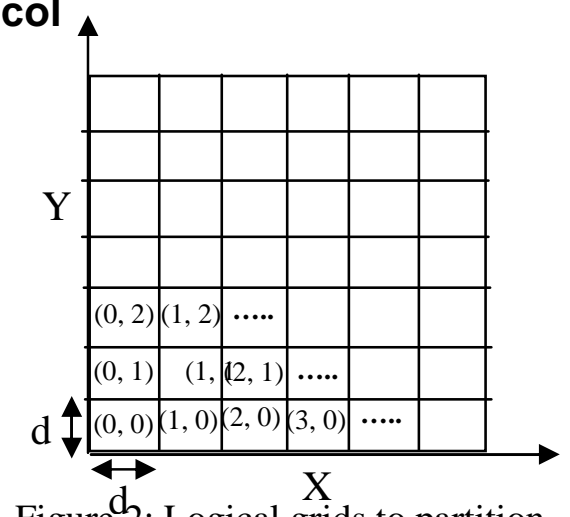

Figure 2 : Logical grids to partition a physical area.

For maintaining the quality of routes, the gateway host of a grid should be the one nearest to the physical center of the grid. Two versions of GeoGRID will be mentioned one called flooding-based and the other called ticket-based.

In the flooding-based version each node serving as a grid gateway within the flooding region will help forwarding geocast messages. In the ticket-based approach, geocast messages are still forwarded by gateway hosts, but not all the gateways in the flooding region will do this job. The concept is similar to that in Ref. [3]. To avoid blind flooding, we will issue a number of tickets; each responsible of carrying one geocast message to the destination region.

\section{2-3 GAMER, Routing-Based Geocast Protocol}

GAMER provides a mesh of paths between the source and the geocast region. When a link breaks due to the changing topology in an ad hoc network, the redundant paths that exist in the mesh can be used. The mesh is created by flooding JOIN-DEMAND (JD) packets within a forwarding zone. Once a node in the geocast region receives a nonduplicate JD packet, it generates a JT packet and unicasts it back to the source following the reverse route taken by the JD packet. All the nodes in the reverse route 
become parts of the mesh. Therfore data packets generated by the source are forwarded by the mesh members within the mesh and flooded within the geocast region. GAMER dynamically changes the density of the mesh by choosing a Forwarding Approach (FA) to keep the network load as low as possible. A source node can choose one approach among CONE, CORRIDOR and FLOOD FAs. Figure 3 shows examples of meshes created with the CONE, CORRIDOR and FLOOD FAs. GAMER adapts its FA to a smaller one if the current FA succeeds, or a larger one if the current FA fails.

\section{3- SIMULATION ENVIRONMENT}

The geocast protocols are implemented in the network simulator NS-2. The simulation area is a $300 \times 600$ meter rectangle. The geocast region is a $150 \times 150$ meter square located in the upper right corner. We put one static node in the center of the geocast region to ensure that at least one node resides in the geocast region to receive the transmitted geocast packets. Each Mobile Node (MN) in the network has a uniform transmission range of 100 meters, and the link bandwidth available for each MN is 2 Mbps. In each 1000 seconds simulation period, the single Constant Bit Rate (CBR) source generates 64-byte geocast data packets.

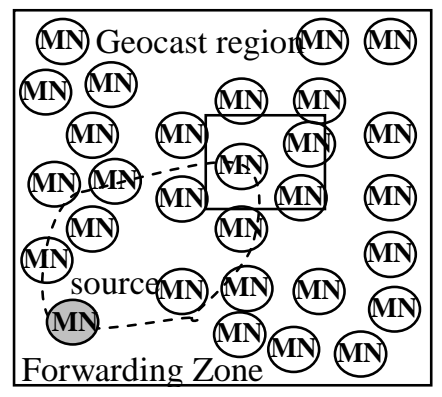

(a)

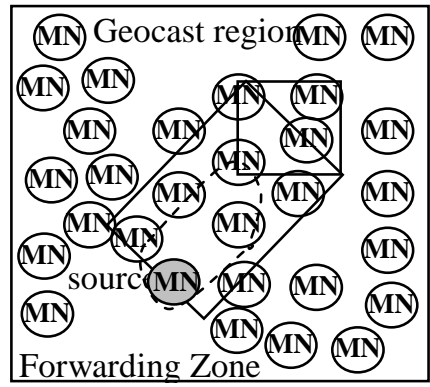

(b)

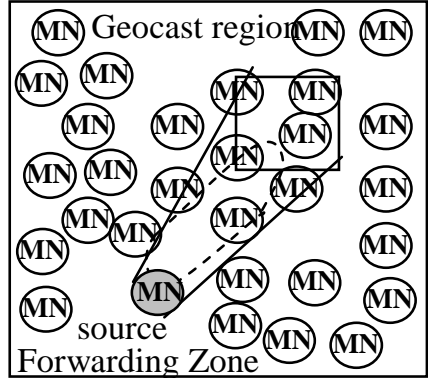

(c)

Figure 3: Examples of meshes created with: (a) FLOOD, (b) CORRIDOR, (c) CONE FAs.

We jitter the scheduling of sending packets by some uniform random amount of time to avoid unnecessary collisions. The nodes move according to the random waypoint mobility model [8]. The random way point mobility model contains pause time between changes in direction and/or speed. Once a Mobile Node begins to move, it stays in one location for a specified pause time. After the specified pause time is elapsed, the MN randomly selects the next destination in the simulation area and chooses a speed uniformly distributed between the minimum speed and maximum speed and travels with a speed $\mathrm{v}$ whose value is uniformly chosen in the interval $(0$, $\left.\mathrm{V}_{\max }\right) . \mathrm{V}_{\max }$ is a parameter that can be set to reflect the degree of mobility. Then, the $\mathrm{MN}$ continues its journey toward the newly selected destination at the chosen speed. As soon as the $\mathrm{MN}$ arrives at the destination, it stays again for the indicated pause time before repeating the process. We initialize the locations and pause times of the MNs with the steady state distribution for the random waypoint mobility model [9].

For the measuring process the metrics are used in the comparison: Throughput, Packet Overhead. The throughput is the amount of data transferred over the period of time expressed in Bytes per second: 


\section{Throughput $=$ Arrival Rate (1- probability of loss).}

and packet overhead is a sum of the number of transmitted packets (data and control) by all nodes in the simulation.

\section{4- SIMULATION RESULTS}

\section{4-1 Parameter Optimization:}

We begin with the LBM protocol: Simulations for different RWP maximum speeds up to $8 \mathrm{~m} / \mathrm{s}$ are performed. The results concerning the packet Delivery Ratio over different speeds were as expected. Generally, when the speed rises the packet Delivery Ratio should decrease. However, in our scenario the average packet Delivery Ratio at $8 \mathrm{~m} / \mathrm{s}$ was still about $0.97 \%$. A reason for this is (even for 80 nodes) the dense network in the scenario. Thus, the choice of the $\delta$ parameter has no significant influence on the packet Delivery Ratio. However, the Packet Overhead increases with a larger $\delta$ parameter.

In figure 4 our simulation shows that the (delta) $\delta=0$ has a lower packet overhead for all speeds (except the speed $=2 \mathrm{~m} / \mathrm{s}$ ) than the higher $\delta$ parameter $(\delta=40,100,160$ unit length). A large count of forwarders in dense networks may show broadcast storm effects. Thus, in dense networks the $\delta$ parameter should be chosen nearby zero.

For the GeoGRID, there are five timers to be set. The Refresh Timer was set to: Refresh Timer $(\mathrm{s})=\frac{\text { Accuracy geo. information }(\mathrm{m})}{\max \cdot \operatorname{Speed}(\mathrm{m} / \mathrm{s})}$. If the geographic information is refreshed more often, then there is no difference due to accuracy of the geo-positioning systems. The calculated value can be seen as a minimum. A larger value, may cause positive impacts concerning power consumption, but there can be negative impacts on the packet Delivery Ratio. The GATE receive timer was set to: GATE receive timer $=2.5 *$ GATE send timer. This means that a node has to miss at least two GATE messages before it starts applying for gateway. BID timer was set to $0.5 \mathrm{~s}$. This seems to be a quite large and thus secure choice. A more accurate choice could be achieved by performing some analytical calculations concerning size of the grid, signal propagation delay, and processing time in a node. The neighbor timer was set to: Neighbor timer $=2 *$ GATE receive timer. Finally the GATE send timer is evaluated for different values. A larger value leads to fewer loads, but there may be a negative impact if the speed rises. Figure 5 shows the average packet Delivery Ratio over the speed. At higher speeds the packet Delivery Ratio decreases. This can be explained by packets losses due to old Gateway elections. However, the GATE send timer has only significant impact on the packet Delivery Ratio for large GATE send timers. The reason for this is the optimal choice of the Refresh Timer. As soon as a node realizes that it has left its grid it sends a retire packet. Thus, in a dense network, as long as the Refresh timer is low enough, a lower GATE send timer has no significant impact on the packet Delivery Ratio. Due to our simulations, a GATE send timer of $0.5 \mathrm{~s}$ seems to be good choice.

\section{4-2 Protocol Implementations:}

This paper classifies the current geocast routing protocols of a MANET into three categories, then simulate a typical geocast routing protocol in each category. We 
performed four case studies designed to highlight the various strengths and weaknesses of the protocols: node density, traffic/congestion, mobility, and a combination case study examining all the three parameters together. LBM is evaluated for floodingbased protocols, GAMER from the routing-based protocol category and chose GeoGRID for cluster-based protocols.

LBM implementation: From our previous simulation for the parameter $\delta$ is set $\square$ for both LBM-box and LBM-step are set to 0. We implement the LBM-box with an adaptive zone.

GAMER implementation: an active GAMER is simulated in this section. In [5] and [6] the authors use source routing in the mesh creation. However, to avoid the overhead caused by storing path information in every JD packet header, nodes maintain local routing tables instead of source routing in our simulation. In other words, the intermediate nodes on a JD packet path maintain routing state, chose 1 second for the JD packet interval, 3 seconds for the mesh-member timeout, and 0.2 seconds for SWITCH-TIMER.

GeoGRID implementation: There are two anomalies that caused GeoGRID code to differ from that suggested in [5] and [6]. First, we restrict all nodes to send exactly one BID packet (which announces a bid to be a gateway) per election rather than one per BID packet received. This modification had no effect on the performance of the protocol other than to reduce overhead. Second, every $300 \mathrm{~ms}$ (not continuously), a node checks whether it has left the grid. We use the simulation code for flooding based GeoGRID, a grid size $\mathrm{d}=\frac{r * \sqrt{2}}{3}$ is used where $\mathrm{r}$ is the transmission radius.

To provide a side-by-side comparison of the geocast protocols, we focus on four case studies. These case studies compare the protocols over a range of network conditions including node densities, node mobility and traffic rates. These cases are outlined in the subsections below.

\section{4-3 Case Study 1 -Effect of Node Density}

To evaluate the core algorithms of the different protocols, case Study 1 compared their performance in a static network using a Null MAC. We varied the number of nodes in the network area from 30 to 120 and fixed the number of sources at $(50,100)$ to decrease the variability in the distance between the source and geocast region. A geocast packet origination rate of 40 packets per second was used, although the use of a Null MAC renders the origination rate irrelevant.

Since the network was static and no collisions were allowed to occur, network conditions that prevented packets from being received rarely occurred. Therefore, packet Delivery Ratio for case Study 1 was almost always 100\%. Thus we've omitted the graph of these results.

Figure 6 shows that LBM-step has the highest packet overhead (worst case) absolutely followed by LBM-box (because in most cases LBM-step results in a larger forwarding zone) and GeoGRID. Packet overhead is increased by increasing the node density.

GAMER is the cheapest in terms of packet overhead. Control packets (if they exist) are sent approximately every second while realistic applications demand that data packets be sent tens and hundreds of times per second. Therefore, those protocols 
that are willing to use control packets to reduce data packets are likely send fewer packets.

It is shown that GeoGRID has a lower packet overhead than LBM-box when 80 or more nodes are present in the network. 80 nodes is the network density where grids start having more than one node in them. That is, 80 nodes is the network density where GeoGRID begins to have a savings in packet transmissions due to the gateways.

\section{4-4 Case Study 2 - Effect of Traffic Rate}

To quantify the effect of congestion on each of the protocols, case Study 2 used the 802.11 MAC in a static network. In this case study, the payload portion of each packet was set at 64 bytes and the rate was varied from 1 packet per second to 80 packets per second. The number of network nodes was set at 80 , roughly the median value from case Study 1. A static network was used for this case study to ensure that the effects of mobility would not interfere with the effects of congestion. As in case Study 1, the source is fixed at $(50,100)$.

Figure 7 shows that LBM-step has the highest packet overhead (worst case) absolutely followed by LBM-box (except for low packet source rates) and GeoGRID. Most of the protocols appear to require a number of transmissions per delivered packet proportional to the packet origination rate. The exception is GAMER, which has periodic control overhead that does not depend upon the packet source rate, giving GAMER a larger control overhead for low packet origination rates. For highly congested networks, GAMER's control packets rise significantly due to the difficulty in making a successful mesh query in a busy network.

Figure 8 shows that for packet origination rates of $30 \mathrm{pkts} / \mathrm{s}$ or below, all protocols have the same Throughput. For higher values of packet source rate GAMER has the best Throughput performance followed by GeoGRID and then followed by LBM-step. It can be seen that LBM-box has the lowest Throughput.

\section{4-5 Case Study 3 - Effect of Mobility}

This case study focuses on the ability of each protocol to react effectively to node mobility in the network. A Null MAC is used in this case study to ensure that no effects from congestion are exist. The packet source rate was set at 40 packets per second. As in case Study 2, the number of network nodes was set at 80 . The range of mean speeds is varied from 1 to 20 meters per second in the simulations.

Figure 9, shows that LBM-step has the highest packet overhead (worst case) absolutely followed by LBM-box, and GeoGRID.

Our simulation shows that a GAMER protocol has the best performance. It transmits the fewest packets for all speeds. All the protocols are basically insensitive to mobility with respect to Packet Overhead.

The overhead of both GeoGRID and GAMER climb slightly due to the extra control overhead required to maintain their state in an unstable network, while both varieties of LBM are statistically unaffected by mobility.

Figure 10 shows that LBM-step and LBM-box have good Throughput in a mobile situation. GAMER protocol has the lowest throughput values as speed increases. GAMER requires that a mesh be maintained in order for packets to be successfully transmitted, which can be broken due to mobility. 


\section{4-6 Case Study 4 - Combined Networks}

In the previous three case studies, one parameter is varied. To complete our evaluation of LBM, GAMER and GeoGRID, the simulation parameters are aggregated into five trials to give an overview of performance at combined conditions. The five trials are designed so that Trial 1 takes a combination of the least severe conditions and Trial 5 takes a combination of the most severe conditions. The specific parameters are shown in Table 1.

Figure 11 shows that the packet overhead is essentially a repetition of the results in the previous three case studies. That is, GAMER uses the least number of packets per delivered packet.

Figure 12 shows that the Throughput for all the protocols is almost equal for Trials 1-3. However, as the network becomes more severe and as GeoGRID is insensitive to mobility and transmission rate factors, GeoGRID protocol has the best Throughput than other protocols, for node densities greater than 80 .

\section{5- CONCLUSION}

In this paper, different types of Geocast routing protocols have been evaluated according to classifying the Geocast protocols into three categories. First simulate (LBM and GeoGRID) in dense scenarios showed in general how to choose parameters. For LBM, the $\delta$ parameter has no effect on the packet delivery ratio. The choice of $\delta=$ 0 has the lowest packet overhead.

For GeoGRID in a dense network, as long as the Refresh timer is low enough, a lower GATE send timer has no significant impact on the packet Delivery Ratio. Due to our simulations, a GATE send timer of $0.5 \mathrm{~s}$ seems to be good choice. The typical geocast routing protocol in each category are simulated. LBM from the flooding based protocols was chosen for evaluation, GAMER from the routing based protocols, and GeoGRID from the cluster-based protocols. NS2 as a simulation environment is used.

Moreover four case studies are performed on each of the protocols to determine their relative strengths and weaknesses: node density, node mobility, node speed, and a combination case study. The conclusions of this paper are : First, GAMER has absolute lowest value for packet overhead. Thus in networks where packet overhead is the only concern, GAMER is preferable. Second, GeoGRID is the most robust, especially in dense networks. GeoGRID does, however, suffer connectivity issues in sparse networks. Finally LBM-box is generally preferable to LBM-step because of the large packet overhead in LBM-step.

\section{6- REFERENCES}

[1] Latiff, L. A. Ali and A. chia-ching Ooi Fisal and N. Vaidya, "Location-Based geocasting and forwarding (LGF) routing protocol in mobile ad hoc networks," industrial conference on telecommunication, Malaysia, July 2005, pp. 536-541.

[2] I. Stojmenovic, A. P. Ruhil and D. K. Lobiyal, "Voronoi diagram and convex hull based geocasting and routing in wireless networks," Eighth IEEE International Symposium on Computers and Communication, 2003. (ISCC 2003). Proceedings. July 2003, pp. 51 - 56 . 
[3] W.-H. Liao, Y.-C. Tseng, K.-L. Lo, and J.-P. Sheu, "GeoGRID: A geocasting protocol for mobile ad hoc networks based on GRID," Journal of Internet Technology, Vol. 2 pp. 23-32, 2000.

[4] C.-Y. Chang, C.-T. Chang, and S.-C. Tu, "Obstacle free geocasting protocols for single/multi-destination short message services in ad hoc networks," Wireless Networks, 9 (2), pp. 143-155, 2003.

[5] J. Boleng, T. Camp, and V. Tolety, "Mesh-based geocast routing protocols in an ad hoc network," IEEE International Parallel \& Distributed Processing Symposium (IPDPS '01), pp. 184-193, San Francisco, CA, April 2001.

[6] T. Camp and Y. Liu, "An adaptive mesh-based protocol for geocast routing," Journal of Parallel and Distributed Computing: Special Issue on Routing in Mobile and Wireless Ad Hoc Networks, 62 (2), pp. 196-213, 2003.

[7] Giordano S, Stojmenovic I., "Position based routing algorithms for ad hoc networks: a taxonomy". In Ad Hoc Wireless Networking, Cheng X, Huang X, Du DZ (eds). Kluwer, Melbourne, Australia, 2004, pp. 103-136.

[8] S Gowrishankar, T G Basavaraju, Subir Kumar Sarkar, "Effect of Random Mobility Models Pattern in Mobile Ad hoc Networks," IJCSNS International Journal of Computer Science and Network Security, Vol. 7 No.6, pp. 160-164, June 2007.

[9] W. Navidi, T. Camp, and N. Bauer, "Improving the accuracy of random waypoint simulations through steady-state initialization," Proc. of the 15th International Conference on Modeling and Simulation (MS 2004), Marina del Rey, California, USA, March 2004, pp. 319-326 .

Table 1: Case study 4-Trial Simulation Parameters.

\begin{tabular}{|c|c|c|c|}
\hline Trial No. & $\begin{array}{c}\text { Number of } \\
\text { Nodes }\end{array}$ & $\begin{array}{c}\text { Avg. Speed } \\
(\mathbf{m} / \mathbf{s e c})\end{array}$ & $\begin{array}{c}\text { Pkt. Src.Rate } \\
\text { (Pkts/Sec) }\end{array}$ \\
\hline 1 & 60 & 1 & 20 \\
\hline 2 & 70 & 5 & 30 \\
\hline 3 & 80 & 10 & 40 \\
\hline 4 & 90 & 15 & 50 \\
\hline 5 & 100 & 20 & 60 \\
\hline
\end{tabular}




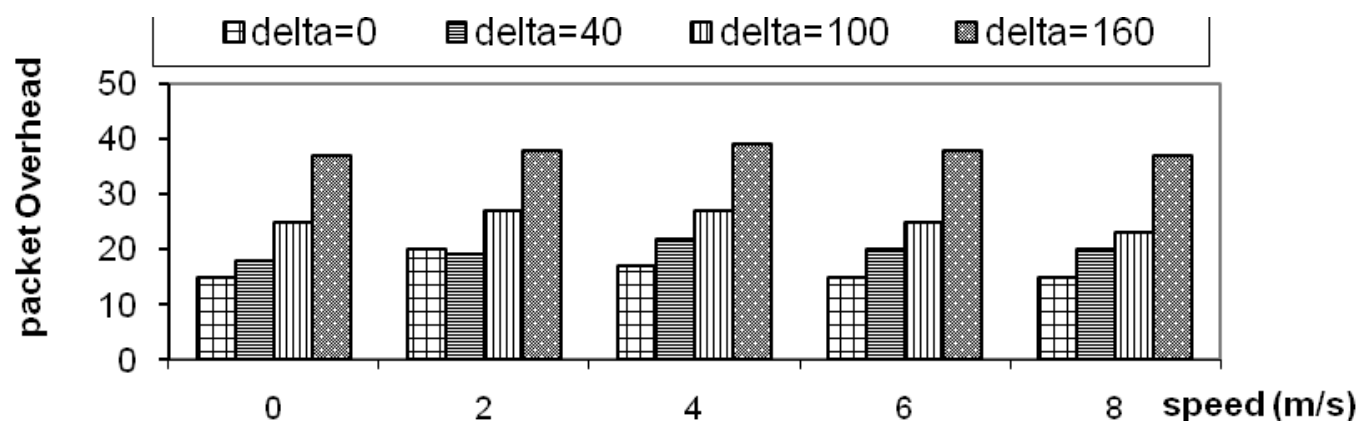

Figure 4: LBM $\delta$ parameter Packet Overhead over speed - 80 nodes.

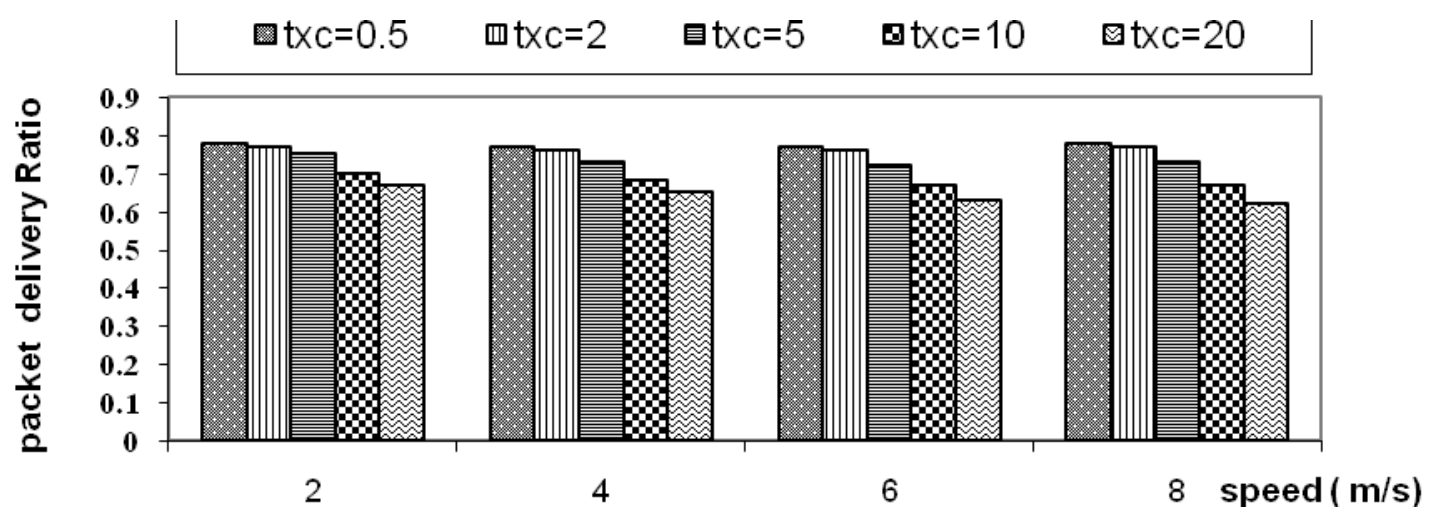

Figure 5: GeoGRID GATE send timer Packet Delivery ratio over speed - 80 nodes.

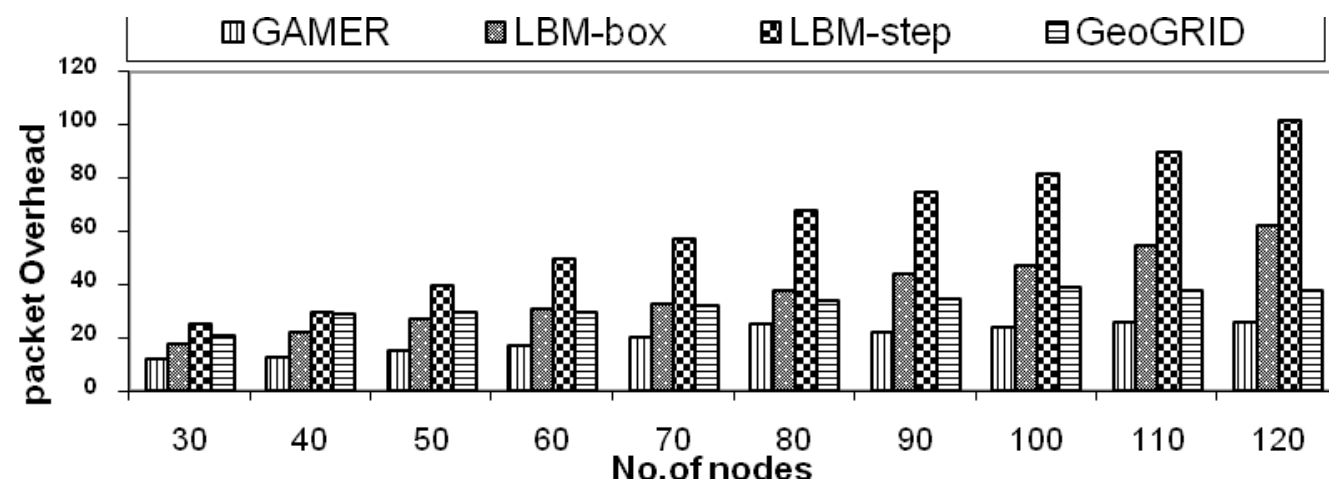

Figure 6: Case Study 1- Packet Overhead versus Number of Network Nodes. 


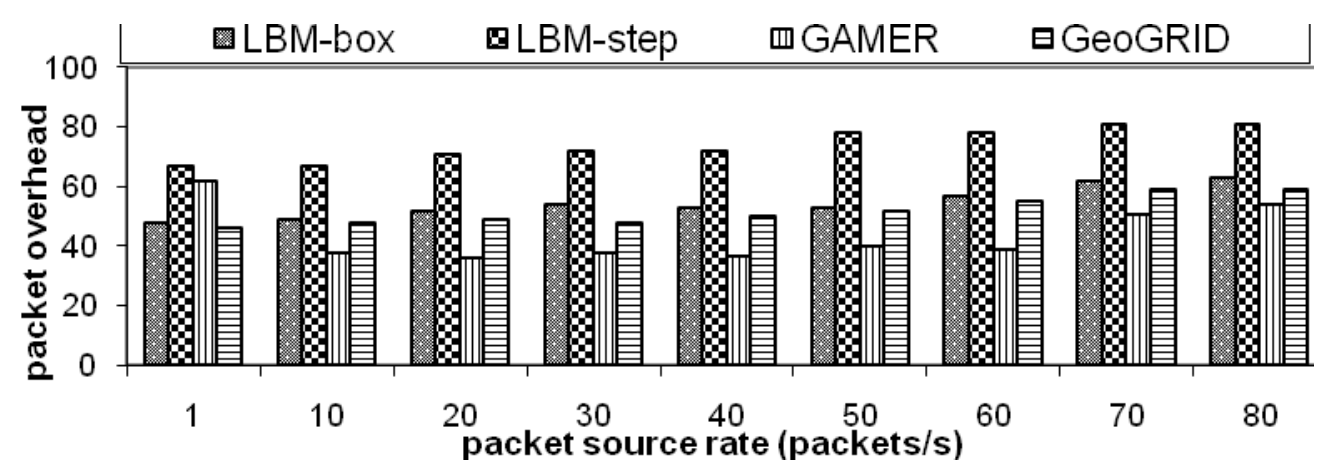

Figure 7: Case Study 2- Packet Overhead versus Packet Origination Rate.

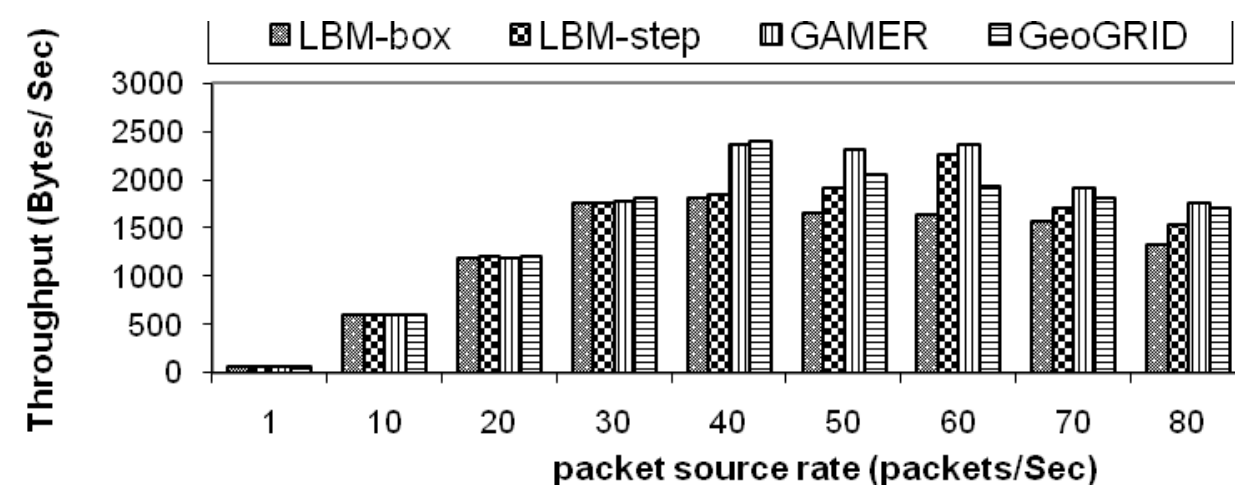

Figure 8: Case study 2- Throughput versus Packet Origination Rate.

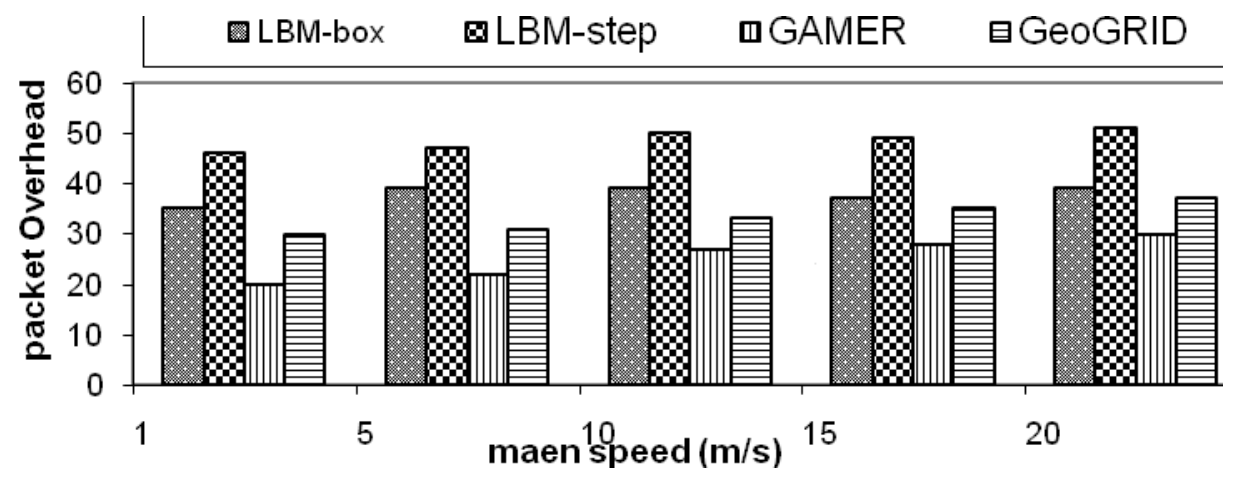

Figure 9: Case study 3- Packet Overhead versus Average Node Speed. 


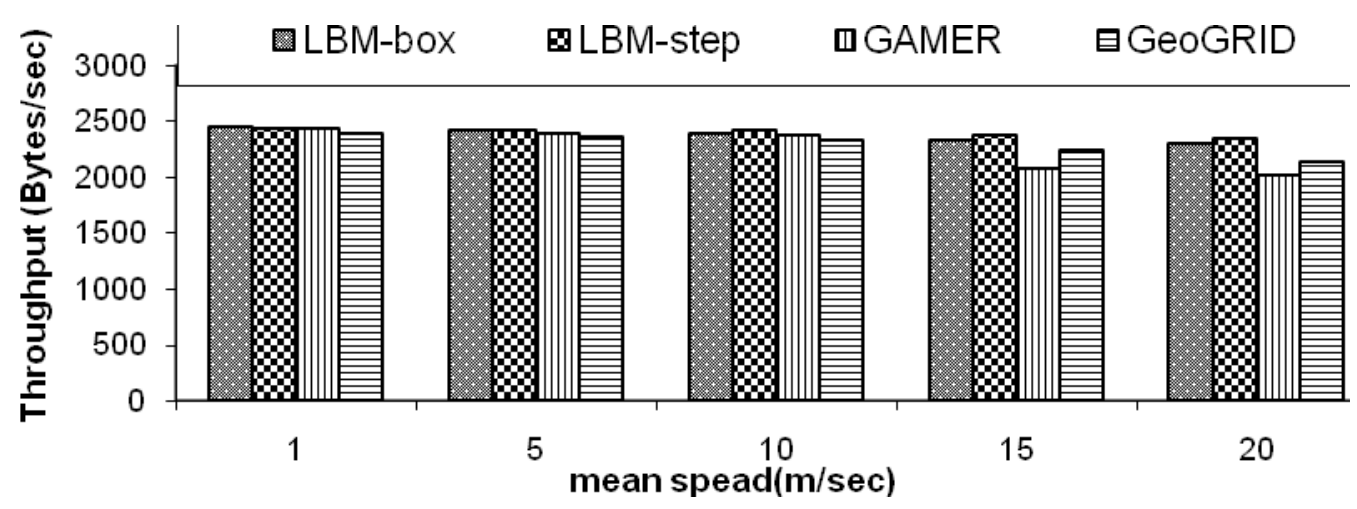

Figure 10: Case study 3- Throughput versus Average Node Speed.

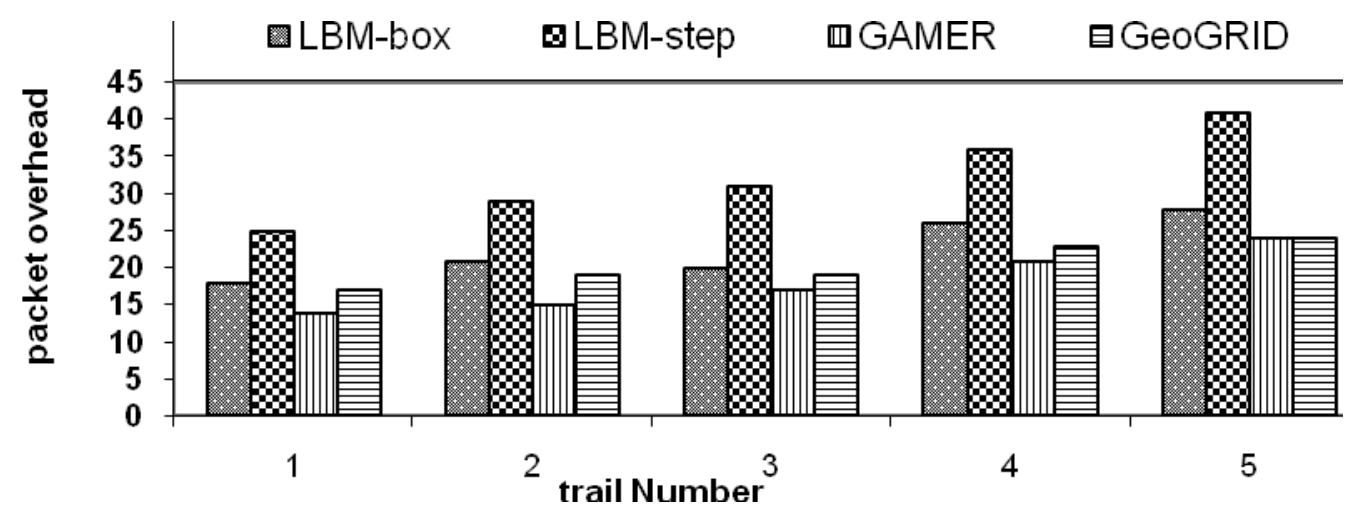

Figure 11: Case study 4- Packet Overhead versus Severity of Network Increase.

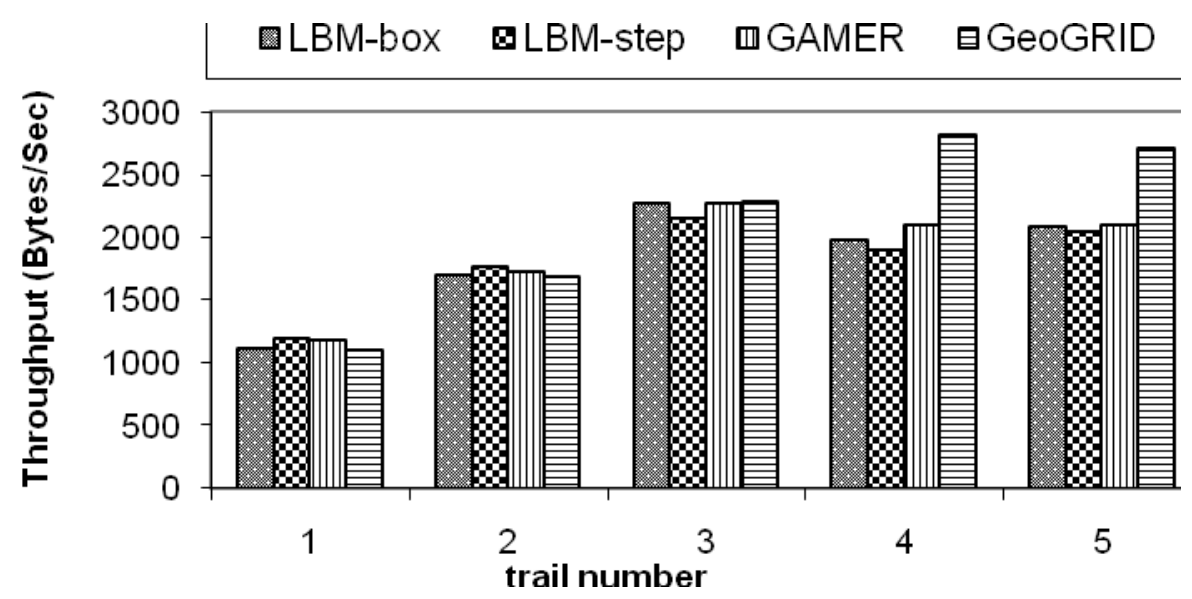

Figure 12: Case study 4: Throughput versus Severity of Network Increase. 


\section{تأثثر البيئة على بروتوكولات تسيير الحيز الجغرافيله}

تتكون شبكة الخدمة الخاصة من مجموعة من المشتركين الذين يتم التواصل بينهم لاسلكيا. تمتاز هذه الثبكات

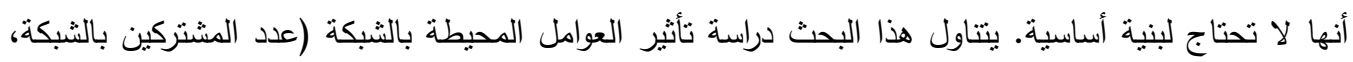

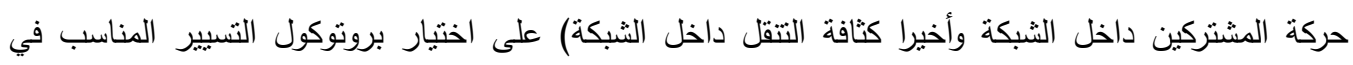

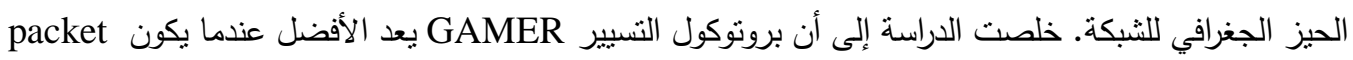
overhead

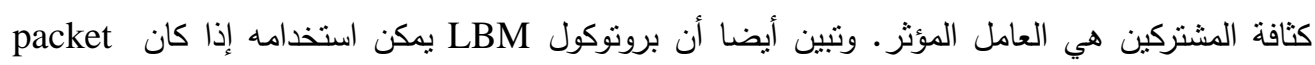

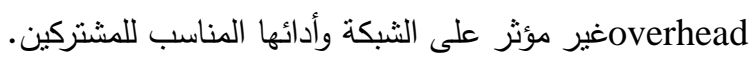

